\title{
Optimum Airport Capacity Utilization under Congestion Management: A Case Study of New York LaGuardia Airport
}

\author{
Loan Le, George Donohue, Karla Hoffman, Chun-Hung Chen \\ Department of Systems Engineering and Operation Research \\ Center for Air Transportation Systems Research \\ George Mason University \\ Fairfax, Virginia 22030
}

\begin{abstract}
(4804 words, including abstract)
Abstract. In the United States, most airports do not place any limitations on airline schedules. At a few major airports, the current scheduling restrictions (mostly administrative measures) have not been sufficiently strict to avoid consistent delays and have raised debates about both the efficiency and the fairness of the allocations. With a forecast 1.1 billion yearly air travelers within the U.S. by 2015, airport expansion and technology enhancement alone are not enough to cope with the competition-driven scheduling practices of the airline industry. The policy legacy needs to change to be consistent with airport capacities.
\end{abstract}

Our research studies how flight schedules might change if airlines had to restrict their schedules to be consistent with runway capacity. To obtain these schedules, we take a novel modeling approach. We model a profit-seeking, single benevolent airline, and develop an airline economic model to simulate its scheduling decisions. This airline is benevolent in the sense that it considers historic pricing at LaGuardia and the associated price-elasticity and attempts to service this population while simultaneously remaining profitable. We explicitly incorporate the relationship between supply and demand through price elasticity, which is estimated by extensive data mining of publicly available databases.

Our case study demonstrates that at Instrument Meterological Conditions ${ }^{1}$ (IMC) runway rates, the market can find profitable flight schedules that reduce substantially the average flight delay while accommodating the current passenger demand at prices consistent with the current competitive market. The IMC rate provides a predictable on-time performance for the identified schedules in all weather conditions. In addition, the reduction of flights through consolidation of low load-factor flights and through aircraft up-gauging alleviate much of the current traffic pressure on high-demand airports. (276 words)

\section{Introduction}

Within the next ten years, FAA Aerospace Forecasts Fiscal Years 2006-2017 [1] predict that there will be as many as 1.1 billion air travelers per year in the U.S. Airports rather than en-route airspace have been identified as the chokepoints creating the major portion of the congestion in the system. An analysis of airport and metropolitan area future

\footnotetext{
${ }^{1}$ IMC generally means that visibility is impaired and runway capacity must be decreased accordingly.
} 
demand and operational capacity [2] reveals that 15 airports will need additional capacity by 2013, and eight more will face capacity limitations by 2020 .

LaGuardia (LGA) airport has a long history of severe congestion. Beginning in 1968, the FAA instituted a High-Density-Rule (HDR) that, for the first time, limited the number of takeoffs/landings by hour or half hour during certain hours of the day. There are only four airports that have such a rule; all other airlines can schedule flights whenever they desire but must ensure that they have the requisite ground facilities (gates, baggage handling, ticket counters, etc) to handle their customers. In late 2000, the FAA provided an additional 300 slots (a 30\% increase in daily operations) into LGA to new entrant carriers or to airlines that fly 70 -seat or less aircraft to small or non-hub airports (see Figure 1). These new slots were labeled "slot exemptions" because they ignored the prior restriction on the total number of slots available at LGA. The result was a significant increase in delays. To overcome this delay problem, a lottery (often called the "slottery") was introduced to reduce the total number of these new slots as a temporary congestion measure to help control the delays. The events of September 11, 2001, and the economic slowdown in mid 2002 reduced demand and congestion, and diverted attention from airport congestion to airport safety. Currently, the growth in air traffic has put substantial pressure on the airport's infrastructure, which has limited possibilities for expansion. Average arrival delay per flight reached 28 minutes in July 2000 and 31 min in July 2005.

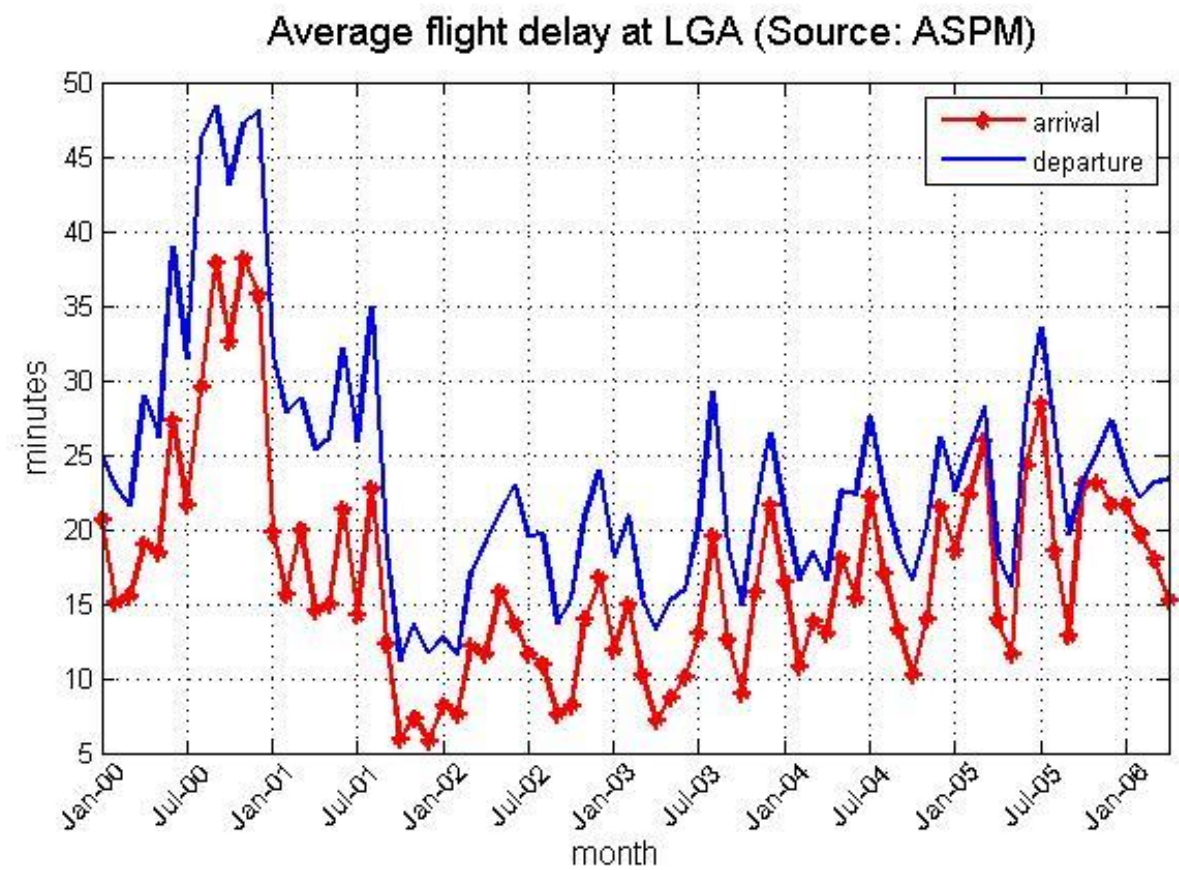

Figure 1. Flight delays at LGA (2000-2005)

At LaGuardia airport, the average number of passenger seats per aircraft in recent years has decreased from 129 seats to 96 seats, see Figure 2. The current scheme of weightbased landing fees and stated customer preference for increased flight frequency produce incentives for airlines to schedule higher frequencies of smaller aircraft to assure their 
continued presence at LaGuardia, even when such slots are not profitable. The provisions associated with the slot exemptions further exacerbated this problem at LGA. Since a small aircraft occupies the same slot as a large one, the throughput per hour of passengers must decline as smaller aircraft are employed. Queues build when airlines continue to add flights to the overall schedule without the addition of new technology or runways. And, by the end of the day, passengers are experiencing long delays and cancelled flights. System wise, regional jets now represent 37 percent of the commercial traffic at the nation's 35 busiest airports, up from 30 percent in 2000 [3]. Actual data for LGA, shows that 50 -seat or less aircraft make up $46 \%$ of the total flights. On the other hand, while having no expansion possibility, LGA has seen the number of operations increase and is now approaching the peak number of scheduled flights experienced in 2000. We can expect that during the summer of 2007 the airport will experience similar delays that will propogate throughout the entire network. .

LGA's annual statistics (Source: BTS)

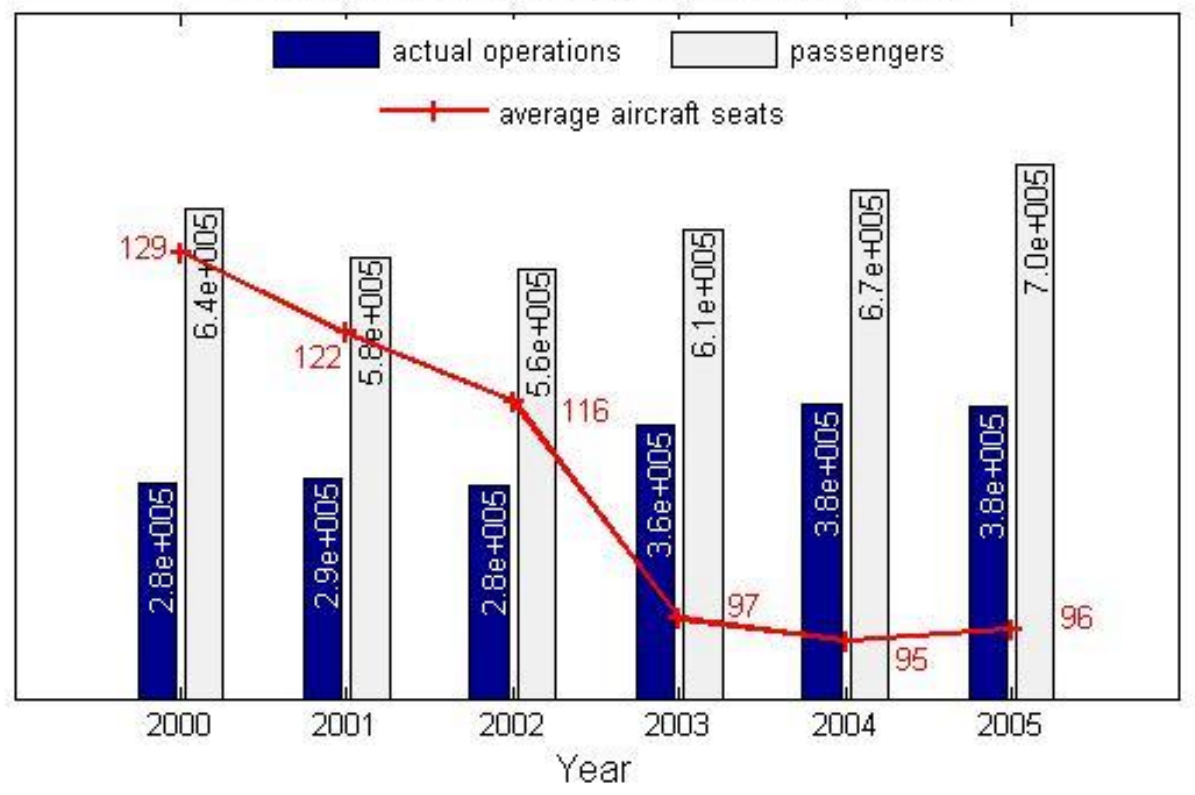

Figure 2. Number of operations, passengers handled and average aircraft seat capacity of at LGA

Other research has shown that delays generated at major airports like LGA, ORD, EWR propagate system wide and that the airlines are not able to absorb these delays in their schedules. There is no technology that can fix delays that are the result of systemic overscheduling. Even if the Next Generation of Air Traffic Control System (NGATS) proposals are deployed, increasing network capacity by reducing aircraft separation standards, the delays will not be reduced because the airlines will respond by increasing their schedules to these high-demand airports. The ability to over-schedule must be brought into alignment with runway capacity. 


\section{Market-based proposals}

Conventional economic wisdom suggests that market-based mechanisms such as congestion pricing and auctions are efficient in allocating scarce resources to the users who value them the most. Both options derive higher fees for peak periods than for offpeak periods, preventing low-value flights from being scheduled in peak periods. In addition, increasing per-flight cost is expected to encourage airlines to up-gauge, and therefore increase passenger throughput.

Congestion pricing consists of charging a flat landing fee based upon demand at a particular time of day. Congestion pricing, as applied to runway allocation would result in the price of an arrival varying by time of day, day of week, and dynamically changing as the demand for operations reacts to these fees. Congestion pricing of transport networks has been common in road traffic. Examples include traditional methods using toll booths such as turnpikes and toll roads, as well as more modern dynamic schemes employing electronic toll collection such as the London congestion charge [4], the Trondheim toll scheme in Norway [5], Singapore's Electronic Road Pricing [6], Highway 407 bypass of Toronto, Ontario, and High-occupancy toll lanes (SR-91, Interstate 15) in California.

Congestion pricing of airport runway access can be considered as a "reactive" measure in the sense that prices are adjusted in response to observed delay levels. A price regulator would set time-based prices for slots and airlines would set their demands accordingly. Comments of The US Department of Justice on congestion pricing [7] pointed out that "a drawback to congestion pricing is the regulator's lack of knowledge about what price to set. A regulator may not have good enough information to allow it to set the right price without frequent experimentation". When set incorrectly, one might experience either over-scheduling (and the resulting delays) or under-scheduling (and the resulting under use) of runways. The airlines may find congestion pricing difficult to manage since it results in both cost uncertainties, and the need to dynamically allocate gates and other ground facilities. Daniel [8], Pels [9], Fan [10], and Schank [11] study the role of congestion pricing of runway slots.

An alternative to congestion pricing is the allocation of slots by auction where the buyer of the slot has complete use of the slot for many years. The buyer has, in essence, leased the right to a given takeoff or landing and can use or re-sell that right for any portion of the lease period. The regulator determines the runway capacity and the airlines then "bid" for the given right for the period specified. Thus, the auction determines the value of each slot "proactively". Auctions have been successfully used for single item sales, corporate procurement, and more complex applications such as telecommunications spectrum allocation with large numbers of interrelated regional licenses [12]. The use of auctions by the Federal Government to allocate scarce resources demonstrates the feasibility of using auctions for complex allocation problems similar to landing-slot allocation. Proposals to allocate airport time slots using market-driven mechanisms such as auctions date back to 1979 with the work of Grether, Issac, and Plot [13] and Rassenti, Smith and Bulfin [14]. European researchers, DotEcon Ltd [15] and National Economic Research 
Associates (NERA) [16], conducted macro-economic analysis to conclude that proper implementation of auctions will result in higher passenger volumes, higher load factors, reallocation of flights to off-peak times or to less congested airports, and lower fares on average. These two works are highly qualitative with illustrative calculations of aggregate statistics. Ball et al. [17] put forward the need for three types of market mechanisms: an auction of long-term leases of arrival and/or departure slots, a secondary market that supports inter-airline exchange of long-term leases and a near-real-time market that allows for the exchange of slots on a particular day of operation.

A series of government-industry strategic simulations or games designed and conducted by member universities of the National Center of Excellence for Aviation Operations Research (NEXTOR) were used to explain to the airline industry and government stakeholders how regulation and market-based allocation mechanisms might be used for runway slot allocation [18]. By simulating real life scenarios, the games allowed interested parties to experience how congestion management and auctions would impact schedules and delays. The results of theses strategic games indicated that the solutions regulators suggested had little impact on delays and congestion. Airlines believed that they were faced with a problem similar to the "Prisoner's Dilemma" whereby if they reduced their flights, they may never be able to recover them. On the other hand, when congestion pricing and auctions were simulated in the same setting, each of these alternatives successfully managed delays because the newly imposed economic costs forced the airlines to reduce the number of departures and landings in their schedule and service passengers with up-gauged aircraft.

\section{Research Problem and Methodology}

We hypothesized that the current congestion situation is caused, in large part, by the existing rules and policies. Specifically, we questioned whether the current grand fathering of slot coupled with an $80 \%$ use-it-or-lose-it requirement, and slot exemptions lead to inefficient use of airport capacity at slot controlled airports. We note that such inefficiency affects both airlines and airports. Faced with projected traffic growth, we wish to determine if alternative rules might (a) better control delays, (b) maintain the profitability of the airline industry, and (c) accommodate current demand at the current competitive prices. We use LaGuardia as our first example because of its importance to the entire network.

In this paper, we explore if there are flight schedules that simultaneously are profitable to the airline industry, accommodate the existing demand at current prices, and reduce congestion. We analyze the schedules we obtained in terms of flight delay, enplanement opportunities, demand profile, average fare, markets served, and aircraft size.

\subsection{Data mining to illustrate inefficient use of runway capacity due to current slot allocation scheme.}

The monthly T-100 Segment Table, compiled by the Bureau of Transportation Statistics (BTS) [19], reports domestic and international operational data by U.S. and foreign air 
carriers. Figure 3 collects six months of data for LGA. The bottom x-axis provides the average aircraft size while the left $y$-axis provides the average load factor. Similarly, the top $\mathrm{x}$-axis and the right $\mathrm{y}$-axis provide the cumulative percentages of the data.

Splitting the charts into four quadrants along the average aircraft size (98 seats) and below $60 \%$ load factors allows us to better understand the observations. The bottom two quadrants are low load-factor flights that are likely unprofitable to the airlines. The left two quadrants relate to low seat capacity flights. Interests of airlines and airports coincide in the upper right quadrant, where the private profitability goal aligns with the public goal of having high enplanement opportunities. The bottom left quadrant is inefficient for both airlines and airports (low load factors coupled with small planes).

Although the majority of flights lie in the upper right hand quadrant, we note that removing the flights in the bottom left quadrant from the system could eliminate all of the delay at LGA. In addition, these flights are not profitable for the airlines. The question then becomes, can one find a schedule that services most (or all) of the current passengers at the times desired at current prices and with aircraft that make these flights profitable?

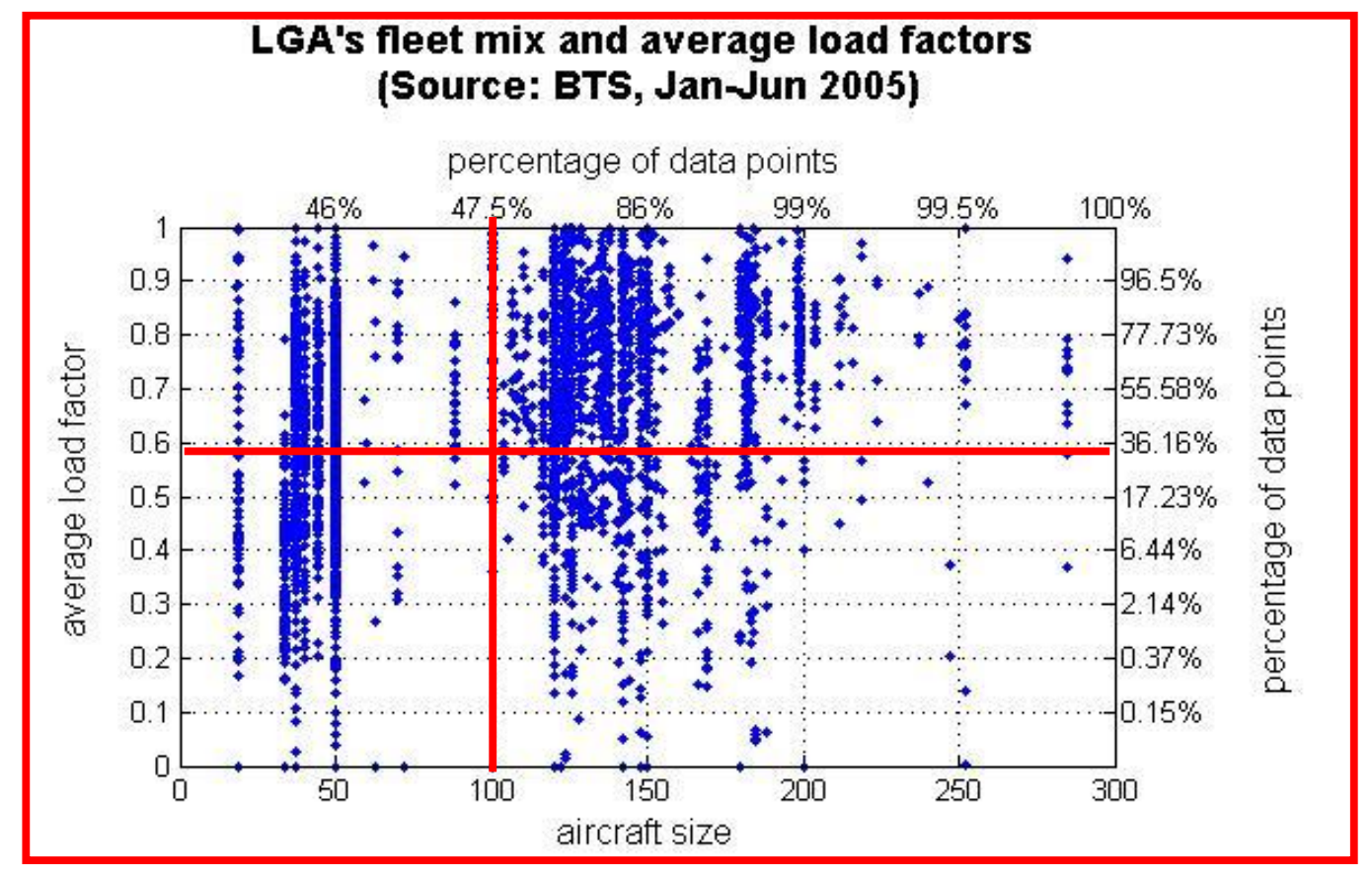

Figure 3. LGA's load factors and aircraft size

Lower left quadrant shows low load factors and small aircraft

3.2. Scheduling models. As stated in the introduction, we take the somewhat unorthodox approach of modeling LaGuardia from the perspective of a benevolent monopoly airline. We are looking to find a schedule that perfectly uses the runway capacity, offers departures and arrivals to LaGuardia consistent with current demand patterns and at current competitive prices. Thus, this airline is looking to serve the region rather than use 
any of its monopolistic power to force a rise in the current prices. To determine such schedules, we use the same algorithms as are used by airlines to determine schedules, and determine the appropriate fleet sizing for that schedule. Using available ticket prices, we estimate demand and supply curves for each market served and we use simulation to determine the delays incurred with any derived schedule.

Flight schedules are determined through the interaction of two processes or models: (i) airlines seek profit-maximizing schedules and (ii) airports maximize enplanement opportunities subject to capacity constraints. The former is called the "sub-problem", and the latter is referred to as the "master problem". In the sub-problems, airlines are modeled aggregately as a single benevolent airline seeking flight schedules for individual markets. The aggregate airline is benevolent in the sense that it reacts to actual price elasticities of demand estimated in a competitive market. Unlike existing airline flight scheduling models that use fare as a parameter, our airline model explicitly accounts for the interaction of demand and supply through price. The airport model in the master problem solves a set packing problem to select the most efficient market schedules. The solution methodology for solving the overall problem is a Dantzig-Wolfe decomposition, where the columns within the master-problem are schedules that are gradually generated based on an announced price vector. As new schedules are presented, the master problem is solved, thereby generating a new price vector. The process continues until an equilibrium is reached, i.e. no new schedules are found that can improve the profitability of the master problem. We note that our methodology applies to airports that have mostly local traffic [20].

\subsubsection{Interaction between demand and supply through price}

In microeconomics, demand and supply interact through price following the generic relationship depicted in Figure 4. The law of demand states that other things remaining equal, the higher the price of a good, the smaller the quantity demanded. This clearly reflects the observations that overcapacity in certain competitive markets have driven airlines to reduce ticket prices to unsustainable levels.

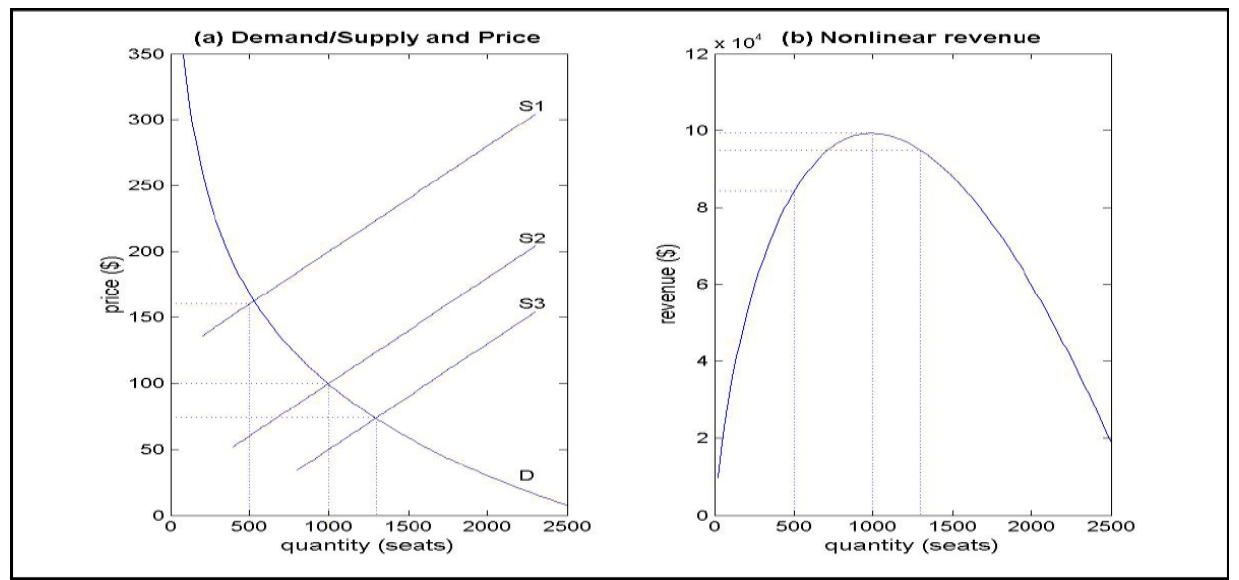

Figure 4. Nonlinear relationship of demand vs. price and the effect on revenues 
A certain fleet mix configuration or ratio corresponds to a supply curve where the movement along the supply curve translates to changes in frequency. Having larger aircraft in the mix shifts the supply curve rightward. Price as a regulator establishes market equilibriums at the intersection points of the demand and supply curves. S1, S2, and S3 in Figure 4 intersect the demand curve D at quantities equal to 500, 1000, and 1300 respectively, where the resulting revenues of S1 and S3 are sub-optimal compared to the revenue of $\mathrm{S} 2$.

Given a market, for all time windows of a particular length, e.g. 15-min, we estimate the convex nonlinear demand curves and the corresponding concave nonlinear revenue functions, where the maximum y-value is the optimal revenue associated with an optimal $\mathrm{x}$-value of supply for a time window. Demand curves of peak periods shift rightward and those of off-peak periods shift leftward. Similarly, revenue curves of peak periods lie on top of those of off-peak periods.

We use nesting revenue functions to model time window interdependency, which manifests through demand spill and recapture among time windows. We first estimate revenues independently for each 15-min time window. We next estimate revenues of time windows of larger granularity, ex. hourly or by time of day (morning, afternoon, evening). It can be reasonably assumed that the sum of demands/revenues of all adjacent 15-min time windows are expected to be constrained by the aggregate, or nested, revenue function of the compounding period, as illustrated by Figure 5. Note that in Figure 5, some 15-min time windows have the same estimates of revenue functions and therefore are superimposed on top of each other.

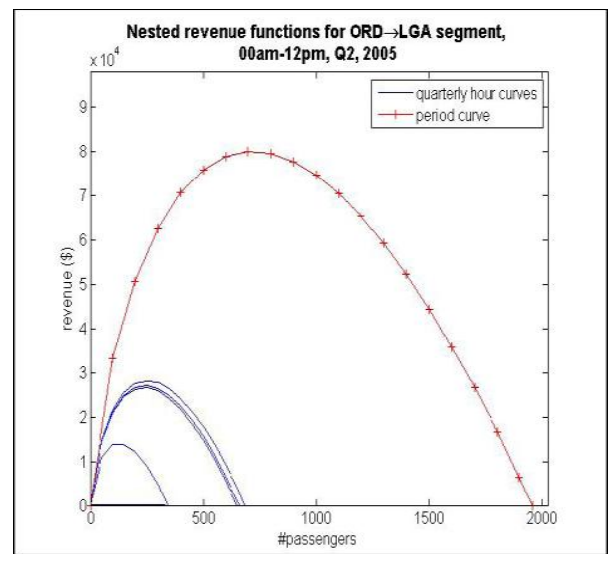

Figure 5: Nesting revenue functions

\subsubsection{Airline scheduling model}

For each market, we build complete schedules for all applicable fleets. At each airport, time of day is partitioned into time windows represented by nodes. Flight arcs are determined by the time needed to fly a particular aircraft type. Similar to Lohatepanont [21], any outgoing arc from a given node is considered to happen after all incoming arcs at that node. There are arcs that allow planes to remain at the airport from one time period 
to the next and there are additional directed ground arcs from the last time window of one day to the first time window of the following day that represent the aircraft that overnight at a given airport.
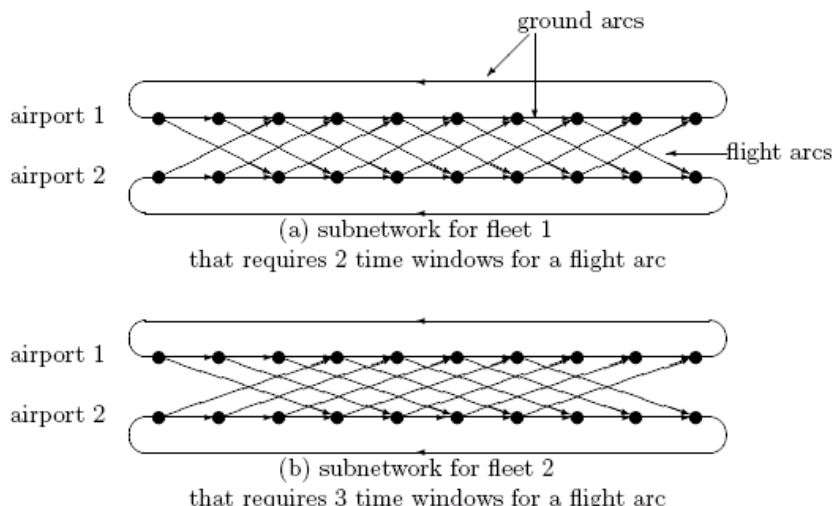

Figure 6: Timeline network example for a city pair having the same time zone.

Assuming concave revenue functions, we define:

Sets:

$T \quad$ time windows

$A^{G} \quad$ ground arcs

$A^{F} \quad$ flight arcs

$K \quad$ fleet types operable for a particular market

$Q(i)$ segment indexes for linear approximation of the revenue function of time window $i \in T$

Parameters:

$S^{k} \quad$ seating capacity of fleet type $k \in K$

$C_{i j}^{k} \quad$ direct operating cost for one flight of fleet type $k \in K$ for $(i, j) \in A^{F}$

$A_{i q} \quad$ linear segment quantities for the revenue function of $i \in T, q \in Q(i)$

$R_{i q} \quad$ linear segment revenues for the revenue function of $i \in T, q \in Q(i)$

$l \quad$ average load factor

Variables:

$x_{i j}^{k} \quad$ number of flights of fleet type $k \in K$ for $(i, j) \in A^{F} \cup A^{G}$

$\lambda_{i q} \quad$ linear segment variables for the revenue function of $i \in T, q \in Q(i)$

Subproblem formulation: 


$$
\max z=\sum_{i \in T} \sum_{q \in Q(i)} R_{i q} \lambda_{i q}-\sum_{(j, i) \in A^{F}} \sum_{k \in K} C_{j i}^{k} x_{j i}
$$

subject to:

$$
\begin{array}{ll}
\sum_{(j, i)} x_{j i}^{k}-\sum_{(i, j)} x_{i, j}^{k}=0 & \forall i \in T, k \in K \\
l \sum_{k \in K} \sum_{(j, i) \in A^{F}} S^{k} x_{j . i}^{k}-\sum_{q \in \sum Q(i)} A_{i q} \lambda_{i q}=0 & \forall i \in T \\
\sum_{i \in \xi(p)} \sum_{q \in Q(i)} A_{i q} \lambda_{i q}-\sum_{r \in Q(p)} A_{p r} \beta_{p r}=0 & \forall p \in P \\
\sum_{i \in \xi(p)} \sum_{q \in Q(i)} R_{i q} \lambda_{i q}-\sum_{r \in Q(p)} R_{p r} \beta_{p r} \leq 0 & \forall p \in P \\
\sum_{q \in Q(i)} \lambda_{i q}=1 & \forall i \in T \\
\sum_{r \in Q(p)} \beta_{p r}=1 & \forall p \in P \\
x \in Z_{+}^{\left|A^{F}\right| \mathrm{x}|K|} \cdot \lambda_{i} \in R_{+}^{|Q(i)|}, \beta_{p}^{|Q(p)|} &
\end{array}
$$

For any time window $i, \sum_{(j, i) \in A} F \sum_{k \in K} C_{j i}^{k} x_{j}^{k}$ in the objective function (1) is the total operating cost of arrivals at $i$. The resulting total capacity $\sum_{k \in K} \sum_{(j, i) \in A}{ }^{F} S^{k} x^{k}$ multiplied by the average factor estimates the number of revenue passengers arriving at $i$. This value is then linearly approximated in (3) as a convex combination of segment endpoints $\left(A_{i q}, R_{i q}\right)$ with $q \in Q(i)$ using non-negative real variables $\lambda_{i q}$. Therefore, $\Sigma_{q \in Q(i)} R_{i q} \lambda_{i q}$ is the piecewise linear approximation of the revenue function of time window $i$. Subtracting the sum of all the cost terms over all flights from the sum of all the revenue terms over all time windows yields the total profit that (1) seeks to maximize. (2) enforces flow balance at each node $i$ in the timeline network, i.e. for each fleet, the number of incoming aircraft is equal to the number of outgoing aircraft of that type.

$\Sigma_{q \in Q(i)} A_{i q} \lambda_{i q}$ is the estimate of realized arrival demand at time window $i$. We note that $i$ can have other substitutable time windows that are all included in a coarser compounding time window $p$, i.e. $i \in E(p)$. Similarly, (4) approximates the aggregate arrival demand of $p$ into a convex combination of segment endpoints $\left(A_{p r}, R_{p r}\right)$ with $r \in Q(p)$ using nonnegative real variables $\beta_{p r}$. (5) states that the sum of revenues of substitutable time windows in $p, \Sigma_{i \in E(p)} \Sigma_{q \in Q(i)} R_{i q} \lambda_{i q}$, is constrained by the revenue of the compounding time window $p, \Sigma_{r \in Q(p)} R_{p r} \beta_{p r}$. (6) and (7) are the sets of convex constraints for $\lambda_{i q}$ and $\beta_{p r}$, respectively.

The solution of a subproblem creates two schedule vectors: the arrival vector $\left\{a_{j}\right\}$ where $a_{j}=\Sigma_{k \in K} \Sigma_{(i, j) \in A} F^{F}{ }_{i j}^{k}$, and the departure vector $\left\{d_{j}\right\}$, where $d_{j}=\Sigma_{k \in K} \Sigma_{(j, i) \in A}{ }^{F} x_{j i}^{k}, j \in T$. These vectors are columns that are added to the master problem. The master problem is then re-solved yielding a new price-vector that is provided to the sub-problem. 


\subsubsection{Airport slot allocation problem}

The master problem at a capacitated airport collects the schedules of individual markets and solves a set packing problem with side constraints to maximize public goals.

Let:

Sets:

$S \quad$ schedule vector indexes

$T \quad$ time window indexes

$M \quad$ market indexes

$S(m) \quad$ column indexes of market m's schedule vectors, $\mathrm{m} \in \mathrm{M}$

Parameters:

$a^{|T| x|S|}$ matrix of arrivals by time window: $a_{i j}$ is the number of arrival flights at time window $i$ in schedule $j$

$d^{|T| x|S|}$ matrix of departures by time window: $d_{i j}$ is the number of departure flights at time window $\mathrm{i}$ in schedule $\mathrm{j}$

$Z_{j} \quad$ coefficient of the schedule vector $j \in S$, determined by the public goal to optimize

$C_{i} \quad$ arrival/departure rates of time window $i \in T$

$G_{i} \quad$ ground capacities in time window $i \in T$

Variables:

$y_{j} \quad$ binary variable, equal to 1 if schedule vector $y_{j}$ is in the optimal solution

Formulation of the master problem:

$$
\begin{array}{ll}
\max _{j \in S} Z_{j} y_{j} & \\
\text { subject to: } & \\
\sum_{j \in S} a_{i j} y_{j} \leq C_{i} & \forall i \in T \\
\sum_{j \in S} d_{i j} y_{j} \leq C_{i} & \forall i \in T \\
\sum_{j \in S(m)} y_{j} \leq 1 & \forall m \in M \\
y \in B^{|\delta|} &
\end{array}
$$

The sets of constraints (9) and (10) reflect airport operational rate constraints. As each market can have many alternative schedules from which at most one schedule can be in the solution, each market has a special-ordered set constraint (11). The objective function maximizes public goals such as:

- Profit, where $Z_{j}$ is the profit of schedule $j$, given by:

$$
\sum_{i \in T} \sum_{q \in Q(i)} R_{i q} \lambda_{i q}-\sum_{(j . i) \in A^{F}} \sum_{k \in K} C_{j i}^{k} x_{j i}^{k}
$$


- Seat throughput, where $Z_{j}$ is the total seat of schedule $j$, given by:

$\sum_{k \in K} \sum_{(j, i,) \in A} S^{k} x_{j i}^{k}$

\section{Results and discussion}

4.1. Parameters and baseline statistics. Our study considered a wide range of scenarios but only one scenario will be discussed in this paper. The scenario we report is one where we first determined the profit-maximizing schedule. We then add a constraint to the model that requires that any schedule be within $90 \%$ of the profit-maximizing schedule and now maximize available seats subject to the model constraints. The full range of scenarios and results can be found in [20]. We have chosen the scenario discussed because it presents a compromise between the two objectives (a) maximizing profit and (b) maximizing throughput.

The data used for this modeling effort include only daily nonstop domestic markets. The following parameters are assumed for all the scenarios in [20]:

- Data sampling period: Q2, 2005

- 67 nonstop domestic markets that have daily schedules to/from LGA

- 45 minutes of minimum turn-around time for all fleets

- $80 \%$ load factor

- Fuel cost: \$2/gallon

- Existing fleets

- Time windows from 6:00am-12:00pm, 12:01pm-17:00pm, 17:01pm-24:00pm are substitutable. However, finer grouping of substitutable time windows can be done to reflect better demand characteristics by time of day for individual markets.

For the sampling period of Q2 2005, ASPM reports traffic data of 275 airports that had nonstop domestic and international flights to/from LGA, and revenue data of 92 domestic markets. We only focus on the 67 domestic markets that have at least one nonstop flight each day during the sampling period, thereby ignoring the 208 other airports that do not have daily flights to LGA.. These 67 domestic markets provide $92.6 \%$ of the total passengers and $94 \%$ actual operations at LGA. Statistics with respect to these 67 markets are collected in Table 1, to be compared our simulated results. The overall statistics are also provided for reference purpose.

\begin{tabular}{|l|l|l|}
\hline Metrics & Study & Overall \\
\hline Nonstop Markets & 67 & 275 \\
Average Daily Nonstop Flights & 1,024 & 1,104 \\
Seats & 98,686 & 101,072 \\
Passengers & 72,845 & 78,675 \\
Average aircraft size & 95 seats/AC & 95 seats/AC \\
Average fare & $\$ 139$ & $\$ 133$ \\
Average flight delay & 18.7 min & $18.6 \mathrm{~min}$ \\
\hline
\end{tabular}


Table 1. Daily average statistics for all flights at LGA, and for the 67 markets used in this study (Source: ASPM Q2, 2005)

\subsection{Results for Airlines Operating at $90 \%$ of Maximum Profit.}

Figure 4 shows the intermediate seat-maximizing solutions within $90 \%$ of optimal profit that (i) are close enough to the baseline to provide a feasible transition solution, and (ii) are reasonably close to the optimal profit curve.

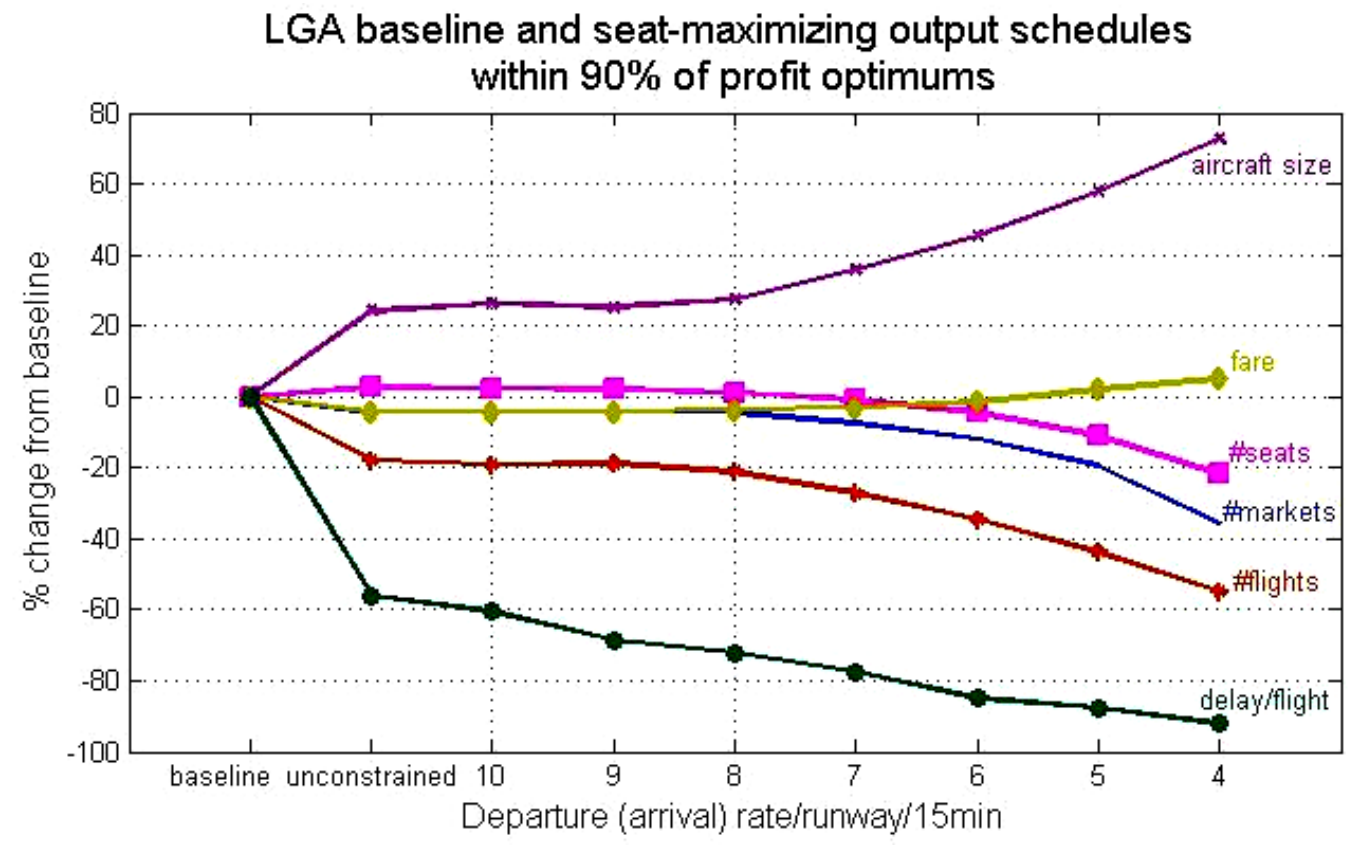

Figure 4. Simulated percentage change in various statistics

At the Instrument Flight Rules ${ }^{2}$ (IFR) rate of 8 arrivals (departures) per runway per 15 minutes, the seat throughputs are slightly higher (1\% increase) than the actual average number of passengers passing through LGA per day during the sampling period. Excluding the three unprofitable markets, the remaining markets remain in this schedule at the IMC rate. All metrics move in the expected direction when runway capacity is further restricted: reduced operations throughput results in increasing aircraft size and average fare while cutting delay.

We review our findings that help answer the research problems stated previously.

Inefficiency due to current slot allocation rules. Actual data for LGA, shows that 50seat or less aircraft make up $46 \%$ of the total flights. Also, 36.2\% of the flights into LGA have load factors of $60 \%$ or less. The main causes for this allocation are: (i) High-

\footnotetext{
${ }^{2}$ IFR rules are more stringent than VFR (Visual Flight Rules) and are in force whenever the Instrument Meteorological Conditions (IMC) specified by the FAA are in effect.
} 
Density-Rule allocations to incumbent airlines who must "use-it-or-lose it" (ii) slot exemptions granted 70-seat or less aircraft, and (iii) weight-based landing fees.

Operational metrics. Table 2 outlines the projected market response with assumptions of $90 \%$ lower bounds on airline optimal profit. Our model predicts positive changes in passengers serviced, aircraft size, and negative changes in flight delay, average fare, and number of flights. The number of markets profitable on a daily schedule stays approximately the same.

\begin{tabular}{|c|c|c|}
\hline Metrics & Baseline & Flight Schedule at 90\% Maximum Profit \\
\hline Number of Markets & 67 & $64 \quad(-4 \%)$ \\
\hline Number of Flights & 1024 & $808 \quad(-21 \%)$ \\
\hline Number of Seats & 96,997 & $98,100 \quad(+1 \%)$ \\
\hline Average Aircraft Size & 95 seats/AC & 121 seats/AC (+27\%) \\
\hline Average Fare & $\$ 139$ & \$134 \\
\hline Average Flight Delay & 19 minutes & 5 minutes (-72\%) \\
\hline
\end{tabular}

Table 2. Projected effects on daily operations at LGA that result from a market-based slot allocation at 8 ops/runway/15 min.

We cannot predict the slot allocation that would result from either auctions or congestion pricing. Past practices of the airlines present ample evidence that airlines are quite costsensitive. When forced to compete and pay for the right to depart and land at a congested airport, they are likely to do the economically efficient thing: use this resource for its best possible purpose. Thus, airlines are likely to continue to service profitable markets and to do so with larger airplanes.

Unprofitable daily markets. This study found only three markets that are not profitable to operate on a daily basis. They are Lebanon-Hanover, NH (LEB), Roanoke Municipal, VA (ROA), and Knoxville, TN (TYS). Under a congestion pricing or auction framework, these markets may have reduced service to La Guardia or have their service moved to a location in the New York area that is not under these market-clearing incentives.

Frequency and delay distribution by time of day. Figure 5 plots the number of flights (arrivals and departures) by their scheduled 15-min time windows and our estimates of flight delay. Note that the output schedule includes only nonstop domestic flights that are profitable on a daily basis. These flights come from 64 airports. Other demands not accounted for include international flights, non-daily and non-scheduled flights that can come from 275 airports having nonstop service to LGA. We stack the other flights on top of the output schedule to approximate the total final demand of this scenario. Time series of average total of actual demand is also plotted for comparison purpose.

We notice that for the $90 \%$ scenario, there is a reduction in the schedule during the offpeak time windows, while the frequency profile approximates relatively well the morning and late evening traffic and results in less delay for arrivals and departures in early evening. 

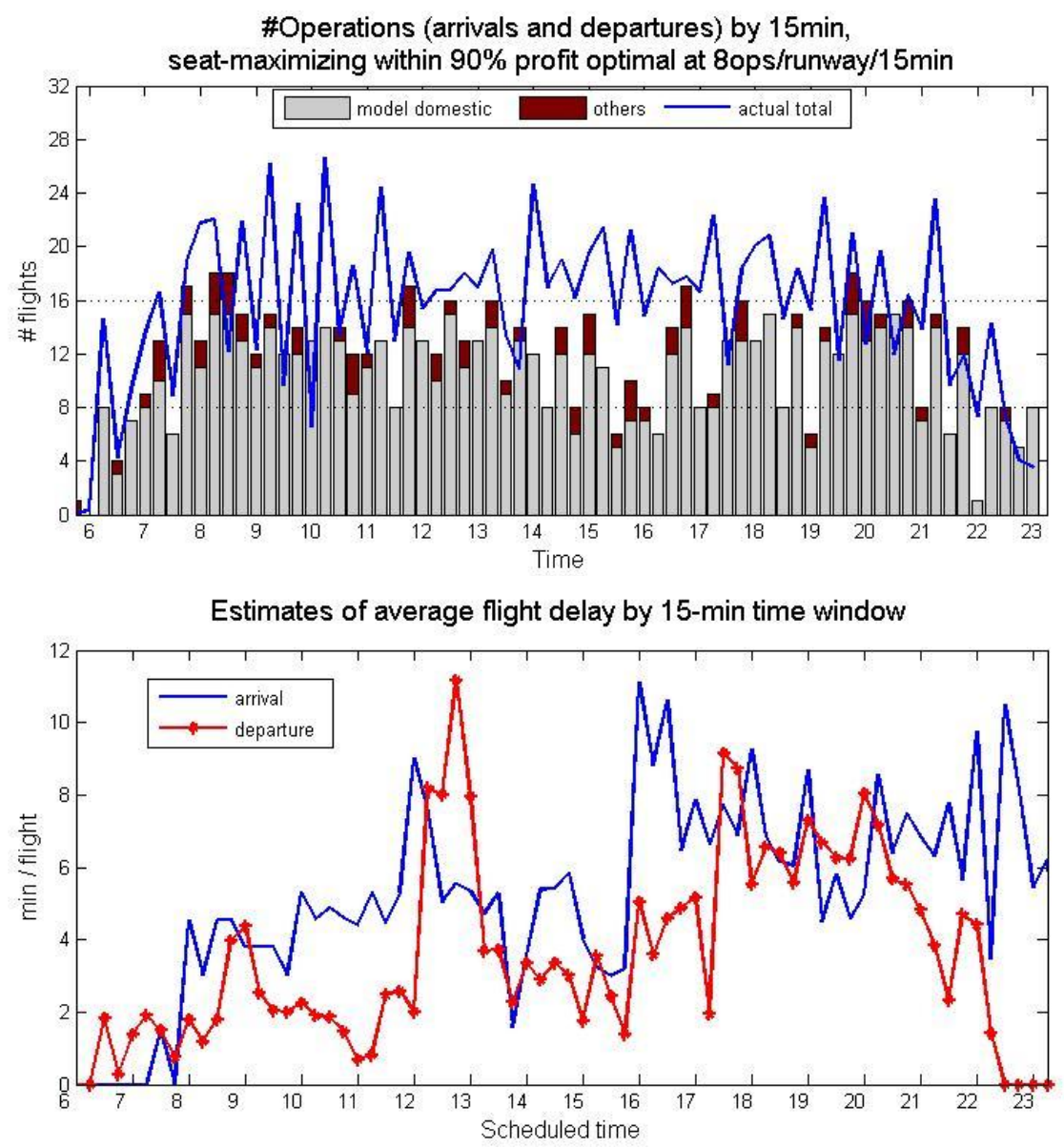

Figure 5. 90\% compromise scenario: frequency and delay profiles

\section{Conclusion}

As airport traffic grows, the need to efficiently use airport facilities becomes essential. Current data shows a large presence of small aircraft and a high number of low loadfactor flights at LGA. A small aircraft occupies the same slot as that of a large one. Thus, when smaller aircraft are substituted for larger aircraft, passenger throughput declines. Thus, when regulators provided slot exemptions to 70-seat or less aircraft, the airlines were forced to compete for the same passenger base but with many more scheduling opportunities. These airlines chose to remain in these markets by decreasing the aircraft size used for each flight. Such over-scheduling resulted in less profitability for all of the airlines serving these markets (as evidenced by low load factors) and simultaneously, resulted in the overloading of the runways. The fact that runway fees are weight-based further encouraged the use of smaller aircraft. Thus, a collection of regulations, fees and administrative policies have forced delays at LaGuardia, propagated delays throughout the network and reduced the overall profitability of the industry. The quandary for the regulator is how to pull back on the current allocation in a manner that is fair and encourages the best use of airport assets. 
Congestion pricing and auctions have emerged as efficient market-based mechanisms for other applications and we believe they can be employed to better allocate runway slots to those who value them the most. Congestion prices result in cost uncertainties for the airline and may be difficult to set properly, but are capable of reacting quickly to changes in demand. Alternatively, auctions allow the airlines to compete for the long-term use of slots, thereby allowing better long-term planning but do not accommodate quick reactions to demand changes. A secondary market for both the selling and exchanging of slots might offset this shortcoming.

In this work, we used an approach that avoids the difficulties of trying to predict the outcomes of either congestion pricing or auctions. We used analytical models consistent with those used by carriers to demonstrate the existence of profitable airline schedules that reduce congestion and accommodate current passenger throughput levels. Two main features characterize the methodology: (a) we model a single benevolent airline instead of individual airlines, and (b) we explicitly account for the inherent demand-supply relation through price. In [20], we analyze multiple scenarios that relax our single benevolent concept. In this paper, we have highlighted one of the most likely scenarios. The results show that at IMC rate, airlines profit-maximizing responses can be expected to find scheduling solutions that offer $70 \%$ decrease in flight delays, $20 \%$ reduction in the number of flights with almost no loss of the markets served or of passenger throughput. This analytic approach should be applicable to other airports or groups of airports facing serious congestion problems. We intend to extend this work to the model a region that has at least three major airports, one of which is a hub airport.

\section{References}

[1] U.S. Department of Transportation, "FAA Aerospace Forecasts Fiscal Years 20062017”, Office of Aviation Policy and Plans, Tech. Rep., March 2005.

[2] FAA and MITRE Corp., "Capacity Needs in the National Airspace System Report", Tech. Rep., June 2004.

[3] FAA, "National Airspace System Operational Evolution Plan 2005-2015", Tech. Rep., February 2005.

[4] T. Litman, "London Congestion Pricing: Implications for Other Cities", Victoria Transport Policy Institute, Tech. Rep., January 10, 2006.

[5] T. Tretvik, Acceptability of Transport Pricing Strategies. Pergamon, Elsevier Ltd., 2003, ch. Urban Road Pricing in Norway: Public Acceptability and Travel Behaviour.

[6] C. Keong, "Road pricing: Singapore's experience" in 3rd Seminar of the IMPRINTEUROPE Thematic Network: "Implementing Reform on Transport Pricing: Constraints and solutions: learning from best practic", Brussels, October 23-24, 2001.

[7] "Comments of the United States Department of Justice on Congestion and Delay Reduction at Chicago O'Hare International Airport”, May 24, 2005.

[8] J. Daniel, "Peak-load-congestion pricing and optimal capacity of large hub airports: With application to the minneapolis-st. paul airport", Ph.D. dissertation, University of Minnesota, 1992.

[9] E. Pels and E. Verhoef, "The economics of airport congestion pricing", Tinbergen Institute Discussion Paper No. 03-083/3, Amsterdam, The Netherlands, Tech. Rep., October 10, 2003. 
[10] T. P. Fan and A. R. Odoni, "The potential of demand management as a short-term means of relieving airport congestion" in Proceedings of EUROCONTROL-FAA Air Traffic Management R\&D Review Seminar, Santa Fe, NM, 2001.

[11] J. Schank, "Solving airside airport congestion: Why peak runway pricing is not working," Journal of Air Transport Management, vol. 11, pp. 417-425, 2005.

[12] P. Milgrom, Putting Auction Theory to Work. Cambridge University Press, 2004.

[13] D. Grether, M. Isaac, and C. Plott, "Alternative methods of allocating airport slots: Performance and evaluation", Pasadena.: Polynomics Research Laboratories, Inc., Tech. Rep., January 1979.

[14] S. Rassenti, V. Smith, and R. Bulfin, "A combinatorial auction mechanism for airport time slot allocation", Bell Journal of Economics, vol. 12, no. 2, pp. 402-417, 1982.

[15] DotEcon Ltd, "Auctioning airport slots: A report for HM treasury and the Department of the Environment, Transport and the Regions", London, Tech. Rep., January 2001.

[16] National Economic Research Associates (NERA), "Study to assess the effects of different slot allocation schemes", London, Tech. Rep., January 2004.

[17] M. O. Ball, G. L. Donohue, and K. Hoffman, "Auctions for the Safe, Efficient and Equitable Allocation of Airspace System Resources", Chapter 17 of Combinatorial Auctions, Cramton, P., Y. Shoham, and R. Steinberg eds. MIT Press, 2005. .

[18] M. Ball, K. Hoffman, G. Donohue, P. Railsback, D.Wang, L. Le, D. Dovell, and A. Mukherjee, "Interim report: The passenger bill of rights game, FAA congestion management game 1 report", NEXTOR Report, Tech. Rep. NR-2005-01, January 2005.

[19] [Online]. Available: http://www.bts.gov

[20] Le, Loan, "Demand Management at Congested Airports: How Far are we from Utopia?", PhD. Dissertation, George Mason University, August 2006, http://catsr.ite.gmu.edu.

[21] M. Lohatepanont, "Airline fleet assignment and schedule design : integrated models and algorithms," Ph.D. dissertation, Massachusetts Institute of Technology, Cambridge, MA, 2002.

\section{Acknowledgment}

This work has been supported in part by NSF under Grant IIS-0325074, by NASA Ames Research Center under Grant NNA05CV26G, and by FAA under Grant 00-G-016."

\section{Short Author Biographies}

Dr. Loan Le conducted this research as part of her Ph.D. program at George Mason University. The work was conducted within the Center for Air Transportation Systems Research (CATSR). She is currently working for American Airlines in Dallas, Texas. Dr. George Donohue is director of CATSR and a Professor of Systems Engineering at GMU. He was formerly the Associate Administrator for Research and Acquisitions at the FAA. Dr. Karla Hoffman is a Professor of Operations Research at GMU with extensive experience in FCC spectrum auctions and airline scheduling and operations. Dr. C.H. Chen is an Associate Professor of Operations Research at GMU with extensive experience in stochastic modeling and simulation of air transportation networks. 